\title{
Is the Motor Impairment in Attention Deficit Hyperactivity Disorder (ADHD) a Co-Occurring Deficit or a Phenotypic Characteristic?
}

\author{
Emily K. Farran ${ }^{1} \cdot$ Aislinn Bowler ${ }^{2} \cdot$ Hana D'Souza $^{3} \cdot$ Leighanne Mayall $^{2} \cdot$ Annette Karmiloff-Smith $^{4} \cdot$ Emma Sumner $^{2}$. \\ Daniel Brady ${ }^{5}$. Elisabeth L. Hill ${ }^{6}$
}

Published online: 4 June 2020

(C) The Author(s) 2020

\begin{abstract}
Objectives Motor difficulties are often reported in individuals with attention deficit hyperactivity disorder (ADHD). The aims of this study are to detail the motor profile of children with ADHD and to determine whether the motor impairment present in a large proportion of children with ADHD represents a phenotypic characteristic of ADHD or a co-occurring deficit.

Methods Participants with ADHD ( $N=51$; age 8 to 15 years) and typically developing (TD) motor matched control children $(N=75$; age 4 to 11 years) completed the largest battery of assessments of motor function that have been used with this population to date, as well as a measure of inhibition as a behavioural measure of ADHD characteristics. Parents/caregivers also completed questionnaires relating to ADHD symptomology and a retrospective report of their child's motor milestone achievement.

Results A motor deficit was observed in $47 \%$ of our ADHD sample. Few relationships were observed between ADHD core characteristics and motor competence. Furthermore, there was an uneven profile of motor performance across different motor tasks, relative to the TD children. Interestingly, it appears that motor milestone achievement is not delayed in ADHD.

Conclusions Our findings suggest that the motor deficit observed in ADHD is not inherent to ADHD. The motor deficit observed in some children with ADHD does not represent a simple delay in development and is not observed in infancy with respect to reaching motor milestones.
\end{abstract}

Keywords Attention deficit hyperactivity disorder $\cdot$ Motor development $\cdot$ Motor milestones

Attention deficit hyperactivity disorder (ADHD) is a common neurodevelopmental disorder characterized by a persistent pattern of inattention and/or hyperactivity-impulsivity (American Psychiatric Association 2013) which affects approximately $5 \%$ of the population (Polanczyk et al. 2007). A co-occurring motor impairment is evident in children with

Emily K. Farran

e.farran@surrey.ac.uk

1 School of Psychology, University of Surrey, Guildford, Surrey GU2 7XH, UK

2 UCL Institute of Education, University College London, London, UK

3 University of Cambridge, Cambridge, UK

4 Birkbeck, University of London, London, UK

5 University of Reading, Reading, UK

6 Goldsmiths, University of London, London, UK
ADHD with approximately 50\% meeting the criteria for Developmental Coordination Disorder (DCD) (BrossardRacine et al. 2012; Pitcher et al. 2003), a disorder characterized by motor skills that are significantly below age-expected levels, despite opportunities to acquire and develop these skills (American Psychiatric Association 2013).

Despite the high prevalence of motor impairments in ADHD, few studies have investigated the motor system in ADHD (see Kaiser et al. 2015 for a review) and it is currently unclear whether a motor deficit is a characteristic of ADHD itself or whether it reflects a co-occurring motor deficit distinct from ADHD symptomology (see Goulardins et al. 2017). Gillberg (2003) categorized those with a dual-diagnosis of ADHD and DCD into a distinct disorder, referred to as deficits in attention, motor control, and perception (DAMP). This is useful at a descriptive level, but it does not address whether motor difficulties are part of the ADHD phenotype or not. Some progress has been made, however, by familial genetic studies. Fliers et al. (2008) suggested from their data of 275 
sibling pairs, at least one of which had a diagnosis of ADHD, that whilst ADHD and motor difficulties have a shared genetic and/or environmental basis, ADHD with a motor impairment appears to represent a separate subgroup compared with ADHD alone.

To address the hypothesis that the motor impairment is part of ADHD symptomology, several studies have investigated the relationship between motor skills and the core diagnostic features of ADHD. Tseng et al. (2004) found that measures of sustained attention and impulse control were good concurrent predictors of fine and gross motor skills in 6- to 11-year-olds with ADHD. Piek et al. (2004) reported strong correlations between motor coordination and inattention, but weaker correlations with executive function $(\mathrm{EF})$ tasks in 6- to 15-yearolds with ADHD. Further, Çak et al. (2018) reported an association between two (fine motor integration and fine manual control) out of eight subdomains of the Bruininks-Oseretsky Test of Motor Proficiency (BOT: Bruininks 1978) and the Conners rating scales (Conners 2003), a measure of ADHD severity. However, their sample was a mixed group of children with ADHD and TD 8- to 11-year-old children, which makes it difficult to identify any ADHD-specific associations. Ziereis and Jansen (2016) found weak evidence that some aspects of EF (visuo-spatial working memory), but not others (inhibition), were related to the aiming and catching, and manual dexterity subdomains of the Movement Assessment Battery for Children second edition (M-ABC 2; Henderson et al. 2007) in 7- to 11-year-olds with ADHD. Fliers et al. (2008) used the Conners rating scales (Conners 2003). They reported that children who presented with ADHD and a motor deficit had equivalent scores compared with those who presented with ADHD only, thus also suggesting that motor impairment is not a marker of severity of ADHD. Given the lack of consistency across studies, Goulardins et al. (2017) concluded that it is not yet clear whether the motor problems in ADHD are a characteristic of ADHD.

Considering the motor profile in those with ADHD, evidence to date suggests that the motor impairment in ADHD does not simply reflect delayed development, rather that there is an uneven profile of impairment across different motor tasks. Standardized motor batteries include the Movement Assessment Battery for Children (M-ABC: Henderson and Sugden 1992) which comprises three subdomains (manual dexterity, aiming and catching, and static and dynamic balance) and the Bruininks-Oseretsky Test of Motor Proficiency (BOT: Bruininks 1978) which comprise eight subdomains (Fine Motor Precision, Fine Motor Integration, manual dexterity, bilateral coordination, balance, running speed and agility, upper limb coordination, and strength) (note that both of these tests have been revised but contain the same subdomains). Studies that have used the M-ABC reported a specific impairment in manual dexterity (Brossard-Racine et al. 2012) or both manual dexterity and aiming and catching
(Pitcher et al. 2003), compared with static and dynamic balance. Studies that have used the BOT reported a deficit in balance and fine motor precision (Tseng et al. 2004) or in balance, fine motor integration, bilateral coordination, running speed and agility, and strength (Çak et al. 2018). Whilst studies show some consistencies, a deficit in manual dexterity is more evident when the $\mathrm{M}-\mathrm{ABC}$ is employed whilst a deficit in balance is more evident when the BOT is employed. This likely reflects differences in task demands; the M-ABC is a tool for screening motor difficulties, whereas the BOT is used to characterize motor impairment and has more subtests that involve similar skills within each subdomain (Johnston and Watter 2006) and thus might be more sensitive to performance variability.

Other studies have used batteries of unstandardised tasks. The majority of these assessed fine motor ability (e.g. pen and paper tracing, mark making, threading beads, putting items on a pegboard, using tweezers) and reported a deficit in children with ADHD relative to TD chronological age-matched controls (Lavasani and Stagnitti 2011; Mokobane et al. 2019; Scharoun et al. 2013). This is broadly consistent with reports of impaired manual dexterity (when measured using the MABC) above. Furthermore, Racine et al. (2008) reviewed evidence for impaired handwriting skill, a practical impact of impaired fine motor skill, in ADHD. Whilst they reported that further research is required, evidence to date suggests that handwriting is impaired in children with ADHD. In addition to fine motor ability, Scharoun et al. (2013) also measured gross motor ability. They demonstrated a deficit in a foot tapping task, relative to TD chronological age-matched controls, but not on gross motor tasks which involved repeatedly tapping metal plates with a rod or manually rolling a matchbox across a table. The authors concluded that the profile of performance reflected task complexity (the more complex tasks lead to impairment). The more complex tasks, which were typically their fine motor tasks, involved motor coordination or goal-directed movements, thus perhaps these tasks drew more heavily on executive function (EF), a skill which is known to be impaired in ADHD.

Beyond motor test batteries, other studies have drawn on evidence that the cerebellum has structural (e.g. reduced inferior posterior vermis: Mostofsky et al. 1998) and functional (e.g. reduced resting state activation in both hemispheres of cerebellar cortex; Kim et al. 2002) atypicalities in ADHD. Motor tasks that are known to be associated with cerebellar activation include balance tasks and finger-thumb tasks (sequentially tapping each finger to the thumb). In contrast to the lack of consensus between the balance subtests of the M-ABC and the BOT above, the four studies that have measured postural sway, arguably a sensitive measures of balance, showed a consistent finding that balance is compromised in both children and adults with ADHD (Bucci et al. 2017; 2014; Hove et al. 2015; Shorer et al. 2012). Hove et al. (2015) also found a 
positive correlation between postural sway and cerebellar volume in adults with ADHD. This supports the theory of balance impairments in ADHD being associated with structural differences in the cerebellum. Interestingly, however, Hove et al. (2015) did not find an association between postural sway and self-reported adult ADHD symptoms. This could suggest that the motor impairment (specifically balance) and cerebellar atypicalities are not inherent to ADHD but represent co-occurring deficits. Further research is required to explore this.

To date, two studies have used the finger-thumb task (Fawcett et al. 1996) with children with ADHD. Both Mostofsky et al. (2006) and Rosch et al. (2013) reported no time difference between 8- and 12-year-olds with ADHD and TD chronological age-matched controls on this task (although Rosch et al. (2013) noted subtle group differences related to variability in the time taken to complete a sequence). This contrasts to the findings of the balance task measures and suggests that the motor profile in ADHD is not solely driven by atypicalities in cerebellar function.

Taken together, the profile of motor impairment in ADHD is yet to be fully established. Evidence to date does not point to a specific deficit in fine or gross motor ability, and impairments extend beyond those that can be attributed to atypical cerebellar function. Whilst Scharoun et al. (2013) discuss task complexity as an explanatory factor for the motor profile observed on their tasks, adopting this explanation cannot explain why postural sway, arguably a task with low cognitive complexity, is impaired in ADHD. Of course, an understanding of the ADHD motor profile is dependent on further knowledge regarding the relationship between the motor impairment in ADHD and core ADHD symptomology. None of the studies above differentiate between those children with ADHD who do and do not have a motor impairment and thus make an implicit assumption that the motor impairment is part of the ADHD phenotype. As such, the findings of the studies above could be argued to be diluted by the inclusion of children with ADHD who do not display a motor impairment. A methodology of splitting the ADHD group into those with and without a severe motor impairment has potential to dramatically increase the precision of our understanding of motor competence in ADHD.

Another factor which is yet to be considered within the ADHD literature is the achievement of motor milestones. In typical development, achieving early motor milestones is vital for infants to interact with and learn from their surroundings (Clearfield 2011; Kretch et al. 2014). Research with atypical populations highlights that late achievement of motor milestones can lead to downstream motor (and other) impairments. For example, children with DCD achieve motor milestones (crawling, standing unassisted, walking unassisted) later than TD children (Sumner et al. 2016). Due to the high prevalence of motor impairments in ADHD, it may also be the case that children with ADHD later identified with a motor impairment achieve milestones later.

The aims of the current study were to (1) determine whether the motor impairment in ADHD relates to the core features of ADHD and (2) determine the ADHD motor profile using the most extensive motor battery that has been employed with children with ADHD to date. To measure ADHD core features, we included a behavioural measure of inhibition as well as a number of parent report standardized questionnaires. To measure motor competence, we used the Bruininks-Oseretsky Test of Motor Proficiency Second Edition Short Form (BOT2SF; Bruininks and Bruininks 2005), a postural sway task and a finger-thumb task. The latter two measures were included because they are known to be associated with cerebellar activation and thus might be impacted by the reported atypicalities in the function of the cerebellum (Kim et al. 2002). We also included a parent report of motor milestone achievement.

\section{Method}

\section{Participants}

Fifty-one children with ADHD (aged 8 to 15 years) were recruited. Three children with ADHD were excluded due to having a co-occurring diagnosis of a neurological condition (partial fetal alcohol syndrome, Tourette's syndrome, or microcephaly). Each of these neurological conditions is associated with motor problems, which could have affected the results. A further two children were excluded because they had taken medication within the $24 \mathrm{~h}$ preceding testing, which can impact motor performance (Kaiser et al. 2015). Finally, three children with ADHD scored $\leq 5$ th percentile on two IQ measures, the British Picture Vocabulary Scale III (BPVS III; Dunn and Dunn 2009) and the Matrices subtest from the British Ability Scales III (BAS III) (Elliot and Smith 2011), and so were excluded because we could not rule out additional learning difficulties which might impact motor performance. The final sample comprised 43 children with ADHD. The ADHD group was recruited by advertising via parent support groups, ADHD publications and newsletters, and social media. All children in the ADHD group had received a formal diagnosis of ADHD from a clinician, which was supported using questionnaire measures. Participants were recruited regardless of their diagnosed ADHD subtype (see Tables 3 and 6 for subtype information). One child with ADHD had a cooccurring diagnosis of DCD. This child was not excluded from the analyses. A further eleven children with ADHD with diagnoses of one or more co-occurring disorders were not excluded because ADHD was their primary diagnosis. Recent research suggests that ADHD might share common early developmental pathways with other disorders, including Autism (see Johnson et al. 2015). Excluding participants with 
co-occurring disorders would both ignore this convergence and would not provide a true representation of the ADHD population. Co-occurring disorders in the current sample were sensory processing disorder $(N=2)$, pervasive developmental disorder $(N=1)$, dyslexia $(N=5)$, autism $(N=3)$, Asperger syndrome $(N=1)$, oppositional defiance disorder $(N=2)$, social communication disorder $(N=1)$, and obsessive compulsive disorder $(N=1)$. All analyses were carried out with these eleven participants excluded and included, and any differences in the patterns of results are reported.

Data from two TD samples are reported. The main TD group were recruited as part of this study and completed the same behavioural measures as the ADHD sample (Table 1). The second TD dataset (Table 2) were provided by Sumner et al. (2016) and had completed the motor milestone questionnaire (other measures were also completed by this group as reported in Sumner et al. 2016). The main TD sample comprised 75 children aged 4 to 11 years who were recruited from primary schools in London, UK. One TD participant scored below the 5th percentile on both the BPVS and BAS Matrices subtest and so was excluded from the group. All TD participants scored in the category of "Average" or "Above Average" with respect to motor ability as measured by the BOT2-SF (Bruininks and Bruininks 2005) and had normal or corrected to normal vision. The final sample size for this group was $N=74$. The TD children were chosen to span the same range of overall motor ability (BOT2-SF) as the ADHD group and so by design were group-matched to the ADHD group by motor ability, $t(111)=0.326, p=0.745$ (see Table 1). As such, many of the TD group were younger than the ADHD group. This methodology of matching by the variable of interest rather than chronological age is not uncommon (e.g. Sumner et al. 2018). To account for the broad range of motor ability and the fact that motor abilities develop at different rates (Clark and Metcalfe 2002) (which would change the profile of motor competences with development), for analyses which involved group comparisons, we split the TD group into three age ranges (TD 4-6 years, TD 7-8 years,
Table 2 TD sample from Sumner et al. (2016)

\begin{tabular}{llc}
\hline & \multicolumn{1}{l}{$\mathrm{TD}(N=34)$} & \\
\cline { 2 - 3 } & $M(S D)$ & Range \\
\hline Age & $9.08(0.94)$ & $7.70-10.74$ \\
Gender (m/f) & $25 / 9$ & \\
BOT2-SF standard score & $56.18(7.25)$ & $43-80$ \\
\hline
\end{tabular}

TD 9-11 years; Table 1). If we had used TD children of the same range in chronological age as the ADHD group, the ADHD group would likely demonstrate impairments across the board thus making it difficult to ascertain profile information, and second, it would fail to take developmental differences in profiles into account because the TD group would be developmentally advanced relative to the ADHD group with reference to motor competence. By using motor ability as our variable of interest, we can determine the profile of motor abilities in children with ADHD relative to the profile of motor abilities of a typical child with the same overall level of motor abilities (note that published standardized data at the subdomain level is not available for the BOT2-SF).

\section{Procedure}

Ethical approval was obtained from UCL Institute of Education. The children were tested individually either in a quiet room at their school, in their home or in the research lab. The order of tests was randomized for each child. For the ADHD participants the session lasted between $1 \mathrm{~h} 15 \mathrm{~min}$ and $2 \mathrm{~h}$ with frequent breaks. For the TD children, testing was completed over four 30-min sessions for children aged 4 to 7 years and in two 1-h sessions for children aged 8 to 11 years. Children were given breaks when needed, and for some 4- and 5-year-olds, some sessions were split further into 15-min sessions in order to maintain motivation and minimize fatigue.

Table 1 TD and ADHD participant information and standardized assessment scores

\begin{tabular}{|c|c|c|c|c|c|c|c|c|}
\hline & \multicolumn{2}{|l|}{ TD $4-6(N=23)$} & \multicolumn{2}{|l|}{ TD $7-8(N=21)$} & \multicolumn{2}{|c|}{ TD 9-11 $(N=30)$} & \multicolumn{2}{|c|}{$\operatorname{ADHD}(N=43)$} \\
\hline & $M(\mathrm{SD})$ & Range & $M(\mathrm{SD})$ & Range & $M(\mathrm{SD})$ & Range & $M(\mathrm{SD})$ & Range \\
\hline Age in years & $5.94(0.76)$ & $4.33-6.96$ & $8.17(0.58)$ & $7.09-8.94$ & $10.08(0.72)$ & $9.09-11.46$ & $11.40(1.89)$ & $8.01-15.63$ \\
\hline Gender (m/f) & $13 / 10$ & & $11 / 10$ & & $14 / 16$ & & $35 / 8$ & \\
\hline BPVS-III standard score & $106.00(11.70)$ & $85-127$ & $105.76(14.67)$ & $74-128$ & $100.07(11.22)$ & $70-121$ & $98.30(11.91)$ & $80-123$ \\
\hline BAS-III Matrices subtest T-score & $43.61(9.099)$ & $29-59$ & $47.81(10.18)$ & $21-73$ & $54.00(13.67)$ & $33-79$ & $45.07(12.88)$ & $20-74$ \\
\hline BOT2-SF standard score & $58.70(6.31)$ & $48-69$ & $58.19(8.17)$ & $46-70$ & $56.13(7.66)$ & $41-69$ & $43.02(8.25)$ & $28-65$ \\
\hline BOT2-SF raw score & $55.17(9.24)$ & $35-70$ & $70.38(70.00)$ & $60-80$ & $74.33(5.28)$ & $62-82$ & $62.42(10.11)$ & $38-80$ \\
\hline
\end{tabular}

BPVS-III British Picture Vocabulary Scale, Third Edition standard score $(M=100, \mathrm{SD}=15), B A S-I I I$ British Ability Scale, third Edition, Matrices subtest T-score $(M=50, \mathrm{SD}=10), \mathrm{BOT} 2-\mathrm{SF}=$ Bruininks-Oseretsky Test of Motor Proficiency Second Edition Short Form, standard total motor score $(M=50, \mathrm{SD}=10)$ 


\section{Measures}

Bruininks-Oseretsky Test of Motor Proficiency Second Edition Short Form (BOT2-SF; Bruininks and Bruininks 2005) The BOT2-SF is a measure of motor skill proficiency for individuals from 4 to 21 years, with strong test-retest reliability (.80 to 0.87 ) and inter-rater reliability $(0.98)$. The test includes 14 items that make up eight subtests: three for fine motor control (fine motor precision, fine motor integration, manual dexterity) and five for gross motor control (bilateral coordination, balance, running speed and agility, upper limb coordination, strength). The test yields a motor composite standard score (mean of 50, standard deviation of 10), in addition to a fine motor and a gross motor raw score. The total testing time for the short form is $15-20 \mathrm{~min}$.

Finger-Thumb Task This motor task (based on Fawcett et al. 1996) involves the experimenter instructing the participant to place both of their hands palm side up on a table, before moving the fingers of their writing hand, touching their thumb to their index finger, then along all their fingers to their little finger, before returning their thumb back along to the index finger. The experimenter demonstrates the task first, before allowing the participant time to practice (until they can complete it). The participant is then instructed to complete two trials of the task, each with six rounds of tapping, as quickly as possible. They are not told how many rounds to complete, only when to stop. Between trials, participants are given a short break and told to "shake your hands" before starting the second trial. The second to the sixth round of tapping for each trial is coded for accuracy. One or more errors in a round would count as one error, with a maximum of five errors per trial and ten errors in total. The task was video recorded and coded offline. Total errors across ten sequences, as well as the summed time to complete ten sequences, were recorded. These variables were used to calculate an inverse efficiency score (IES: response time/(1 - proportion of errors)).

Wii Balance Board (WBB) The WBB is a valid and reliable tool for measuring postural control (Clark et al. 2010). This was used as our measure of balance. Participants stood with their feet together, without shoes, in the middle of the board. They were instructed to stand as still as possible for four $20 \mathrm{~s}$ sessions, either with their eyes open or closed (the order of which was interleaved and counterbalanced). The WBB was connected to a laptop over Bluetooth. Postural sway was quantified as the path length (in $\mathrm{cm}$ ) of the centre of pressure ( $\mathrm{CoP}$, defined as "the location on the supporting surface where the resultant vertical force vector would act if it could be considered to have a single point of application" (p. 3, Benda et al. 1994). The CoP path length was calculated using formulae from Leach et al. (2014). To account for the anticipated noisier data from the WBB due to the low weight of the younger participants, a wavelet filter was applied. The filter and the threshold used are as described in Flatters et al. (2014).

Inhibition (Go/No Go Task) The Go/No Go task (see Purser et al. 2015) provided an empirical measure of ADHD characteristics. The task was programmed in MATLAB. Participants viewed a pseudo-random series of red, green, blue, and yellow solid 5-cm-diameter circles, presented on a laptop and were instructed to press the space bar as quickly as possible when they saw each circle. If the circle was red, the participant was told to refrain from pressing the space bar. If the space bar was pressed on viewing a red circle, a buzzing "error" noise was heard (via headphones) and the circle disappeared. Each circle disappeared after $2 \mathrm{~s}$ if the space bar was not pressed. Red trials constituted $1 / 4$ of trials. There were two blocks of 64 experimental trials, preceded by eight practice trials, with a break between blocks. The total number of commission errors (i.e. the number of times they pressed the space bar for a red circle) was measured. Response time (RT) variability was measured as the standard deviation of RT for correct hits (see Epstein et al. 2011).

Conners' Parent Rating Scale - Revised Long Form (CPRS-R:L: Conners 1997) CPRS-R:L can be used for children from 3 to 17 years. Parents rate their children's behaviours over the last month on 80 items, on a four point scale from "Not True At All (Never)" to "Very Much True (Very Often)". It provides 14 subscales, including an ADHD Index (comprised of questions on both Inhibition and Hyperactivity). This contains the best-suited items for distinguishing ADHD children from children without ADHD and is presented in the current study. Scores on subscales that are one standard deviation above the mean of 50 (i.e. scores above 60 ) are considered to be in the clinical range. The questionnaire has strong internal reliability (0.75-0.94, Conners et al. 1998) and the ADHD Index test-retest reliability is 0.72 . The ADHD Index also provides an indication of ADHD subtype according to DSM IV criteria in the following categories: ADHD inattentive, ADHD hyperactive-impulsive, and ADHD combined. Given that the response items used to determine subtype only partially overlap with those which comprise the ADHD Index score, it is also possible to score in the clinical range for the ADHD Index, but not meet criteria for either the categories listed above.

Child Behaviour Checklist (CBCL: Achenbach and Rescorla 2001) The CBCL is a widely used instrument for children between 6 and 18 years. Parents rate their child's behaviour now and within the past 6 months on 113 items. The behaviour is scored on a 3-point Likert scale from 0 (not true) to 2 (true/ often true). Items are organized into a number of scales. The attention deficit/hyperactivity scale is based on the DSM (Diagnostic and Statistical Manual of Mental Disorders) criteria for ADHD and was used in the present study. T- 
scores above 65 are in the clinical range. The attention deficit/ hyperactivity scale has a strong internal reliability $(0.84)$ and test-retest reliability $(0.93)$.

Strengths and Difficulties Questionnaire (SDQ: Goodman 1997) The parent report version of the SDQ comprises 25 items. Items are rated as: "Not True", "Somewhat True", or "Certainly True". Items are organized into five subscales; given the focus of the present study (on ADHD), the hyperactivity subscale scores were used. A Hyperactivity score higher than 5 is considered to be "Raised", with a score of 8 or more considered to be "High". The measure has a good internal consistency (.73), and test-retest reliability $(0.62$, Goodman 2001).

Motor Milestones Questionnaire (Developed by Sumner et al. 2016, and Based on Brouwer et al. 2006) Parents were presented with a list of 12 motor milestones (e.g. stand without assistance) and were asked to report the child's age (in months) that these milestones were reached, plus any further comments about the motor milestone.

IQ Measures Participants completed the British Picture Vocabulary Scale III (BPVS; Dunn and Dunn 2009), a measure of receptive vocabulary, and the Matrices subtest of the British Ability Scales III (BAS) (Elliot and Smith 2011), a measure of visuo-spatial reasoning.

\section{Data Analyses}

To assess ADHD core characteristics, parent report measures of ADHD characteristics were scored and coded categorically to determine whether participants with ADHD met the cut-off for ADHD or not. Performance on the Go/No Go task, our behavioural measure of ADHD characteristics, was analysed using one factor ANOVAs with Group (TD 4-6 years, TD 7-8 years, TD 9-11 years, ADHD) as the between-participant factor. The two dependent variables for the Go/No Go task were commission errors and RT variability. We predicted that the ADHD group would all meet the cut-off for ADHD and would demonstrate a relatively high number of commission errors and higher RT variability compared with at least the TD 9-11 years group.

To determine whether the motor impairment in ADHD relates to the core features of ADHD (aim 1), first associations between motor performance and ADHD core characteristics were analysed using correlational analyses. Correlations were carried out for the ADHD group and TD group (treated as one group) separately. In light of the implications of relying on associational designs (an association does not imply causation), this approach was coupled with our second approach. That is, we categorically divided our ADHD group using the zones of the BOT2-SF (Bruininks and Bruininks 2005) into those with a motor ability score which indicates impairment $(<16$ th percentile ADHD-L) and those with motor performance within the normal range $(\geq 16$ th percentile ADHD-H). We compared the ADHD core characteristics and motor scores between the ADHD-L and ADHD-H groups using independent samples $t$ tests. On account of the lack of consistency in the literature regarding whether or not a motor impairment is part of the ADHD phenotype (Goulardins et al. 2017), we cannot make predictions for aim 1 . If a motor impairment is inherent to ADHD, this predicts an association between ADHD core characteristics and motor performance, as well as group differences (ADHD-L > ADHD-H) for both motor impairment and ADHD characteristics. If a motor impairment in ADHD instead represents a subgroup of children, this predicts no such association and that the ADHD-L group will differentiate from the ADHD-H group with respect to motor skills, but not ADHD core characteristics.

To determine the ADHD motor profile (aim 2), MANOVA was carried out on the eight subdomains of the BOT2-SF (fine motor precision, fine motor integration, manual dexterity, bilateral coordination, balance, running speed and agility, upper limb coordination, strength) with Group as a between-participant variable (TD 4-6, TD 7-8, TD 9-11, ADHD-L, ADHD-H). For the motor milestone and finger-thumb IES-dependent variables, onefactor ANOVAs were carried out with Group as a betweenparticipant variable (TD 4-6, TD 7-8, TD 9-11, ADHD-L, ADHD-H), whilst a two-factor ANOVA with an additional factor of eyes open vs. eyes closed was carried out for postural sway. Postural sway had a dependent variable of path length (in $\mathrm{cm}$ ) of the centre of pressure $(\mathrm{CoP})$. Our method of categorizing the participants with ADHD into two groups for these analyses represents a significant departure from the methodologies used in the literature to date, which risked diluting findings by the inclusion of children with ADHD who did not display a motor impairment. We predicted an uneven profile of impairments and delayed motor milestone achievement in at least the ADHD-L group, with stronger impairment on balance measures and possibly manual dexterity, relative to other motor tasks and fingerthumb performance. Furthermore, if the motor profile of the ADHD-L group is specific to this group, this supports a hypothesis that the two ADHD groups are categorically different with respect to motor competence and that children with $\mathrm{ADHD}$ and a severe motor impairment represent a subgroup of ADHD. In contrast, if the two ADHD groups present with similar, atypical motor profiles, this supports the hypothesis that the two ADHD groups fall on a continuum of motor performance and that there is a disorder-specific motor profile for ADHD.

\section{Results}

\section{ADHD Core Characteristics}

Parental Questionnaires Parental questionnaire data for the ADHD group are reported in Table 3. One parent did not 
complete the SDQ, and four parents did not complete the CBCL. All ADHD participants met the criterion of having a CPRS-R:L ADHD Index score above 60. Thirty-two out of 39 (82\%) ADHD participants met the criterion of having a CBCL attention deficit/hyperactivity problem T-score at or above 65 . Forty-one out of 42 (99\%) ADHD participants met the criterion of having SDQ hyperactivity scores at or above 5. In summary, all participants met criteria for ADHD on at least one of the questionnaire measures. This supports their clinical diagnosis of ADHD.

Go/No Go Commission errors on the Go/No Go task and RT variability represent a behavioural measure of the core ADHD deficit of inhibition (Nigg 1999; although see Nigg et al. 2005) and a key marker of risk for ADHD symptoms (Tye et al. 2016) respectively. The data from three TD participants are missing, due to technical failure, whilst a further three TD participants did not attempt the task. ANOVA was carried out on each of these two dependent variables with Group (TD 46 years, TD 7-8 years, TD 9-11 years, ADHD) as a betweenparticipant factor. This demonstrated a main effect of group for each DV (commission errors, $\mathrm{F}(3,107=3.79, p=.013$, $\eta_{\mathrm{p}}{ }^{2}=0.096$; RT variability, $\mathrm{F}(3,107)=3.91, p=0.011$, $\left.\eta_{\mathrm{p}}{ }^{2}=0.99\right)$. Tukey paired comparisons demonstrated that this was due to poorer performance (mean (SD), 5.98 (5.37)) and higher RT variability (mean (SD), $0.21(0.09)$ ) in the ADHD group than the TD 9-11 group (commission errors mean (SD), 2.73 (3.34); RT variability mean (SD), $0.16(0.05)$ ) (commission errors, $p=0.008$; RT variability, $p=0.014$ ), but similar performance to the TD $4-6$ (commission errors mean (SD), 3.88 (3.67); RT variability mean (SD), $0.22(0.07))$ and TD 7-8 (commission errors mean (SD), 3.91 (2.57); RT variability mean (SD), $0.19(0.06)$ ) groups ( $p>0.05$ for all) (see Figs. 1 and 2). The TD 9-11 group did not differ significantly in cognitive ability from the ADHD group (BPVS raw score, $p=0.75$; BAS matrices ability score, $p=0.06$ ) and thus represent an approximate cognitive ability match. Thus, this again supports the clinical diagnosis of ADHD for this group.

\section{Associations between Motor Performance and ADHD Core Characteristics}

On account of high correlations between the CPRS-R:L ADHD Index and the CBCL attention deficit/hyperactivity $(r=0.57, p<0.001, N=39), \mathrm{CBCL}$ attention deficit/ hyperactivity was not entered into correlation matrices. Correlational analyses were carried out for the ADHD group between four measures of ADHD core characteristics (two behavioural and two parent reports) and eight motor measures (five concurrent and three retrospective). For the TD group (treated as one group), correlational analyses were carried out between the two behavioural measures of ADHD core characteristics and the five concurrent motor measures.

The two behavioural measures of ADHD core characteristics were Go/No Go commission errors and RT variability, whilst the two parent report measures were CPRS-R:L ADHD Index and SDQ hyperactivity index. The five concurrent motor measures were two measures from the BOT2-SF (fine motor score and gross motor score) and three cerebellum measures (postural sway eyes open, postural sway eyes closed, finger-thumb IES). For the concurrent motor measures, partial correlations were carried out, controlling for variance associated with chronological age. The retrospective motor measures were three motor milestone measures (sitting without support, hands and knees crawling, walking without support). These motor milestone measures were chosen because they all signified unsupported motor competences and thus would not be impacted by variation in parental scaffolding. This constitutes 32 correlations for the ADHD group, and thus, the Bonferroni-corrected critical alpha is $p \leq 0.00156$ and ten correlations for the TD group, and thus a Bonferroni-corrected critical alpha of $p \leq 0.005$. The TD group demonstrated no significant correlations (controlling for chronological age) between the behavioural measures of ADHD characteristics (derived from the Go/No GO task) and the concurrent motor measures, $p>0.00625$ for all; range, $p=$ 0.046 to $p=0.90$ ). Correlations for the ADHD group are also largely non-significant. These are reported according to

Table 3 Parental Questionnaire scores: ADHD group

\begin{tabular}{lccl}
\hline & $M(S D)$ & Range & Subtype \\
\hline $\begin{array}{l}\text { CPRS-R:L ADHD Index } \\
(N=43)\end{array}$ & $77.81(7.86)$ & $61-90$ & $\begin{array}{l}\text { Inattentive: } N=10 \\
\text { Hyperactive-impulsive: } N=5 \\
\text { Combined: } N=13\end{array}$ \\
*Meets neither subtype criteria: $N=15$ \\
SDQ Hyperactivity $(N=42)$ & & & NA \\
CBCL attention deficit/hyperactivity $(N=39)$ & $8.69(1.44)$ & $3-10$ & NA \\
\hline
\end{tabular}

CPRS-R:L Conners Parent Rating Scale-Revised Long version, $S D Q$ strengths and difficulties questionnaire, $C B C L$ child behaviour checklist. *Note that the response items used to determine CPRS-R:L subtype only partially overlap with those which comprise the ADHD Index score. As such, it is possible to score in the clinical range for the ADHD Index, but not meet criteria for either subtype 


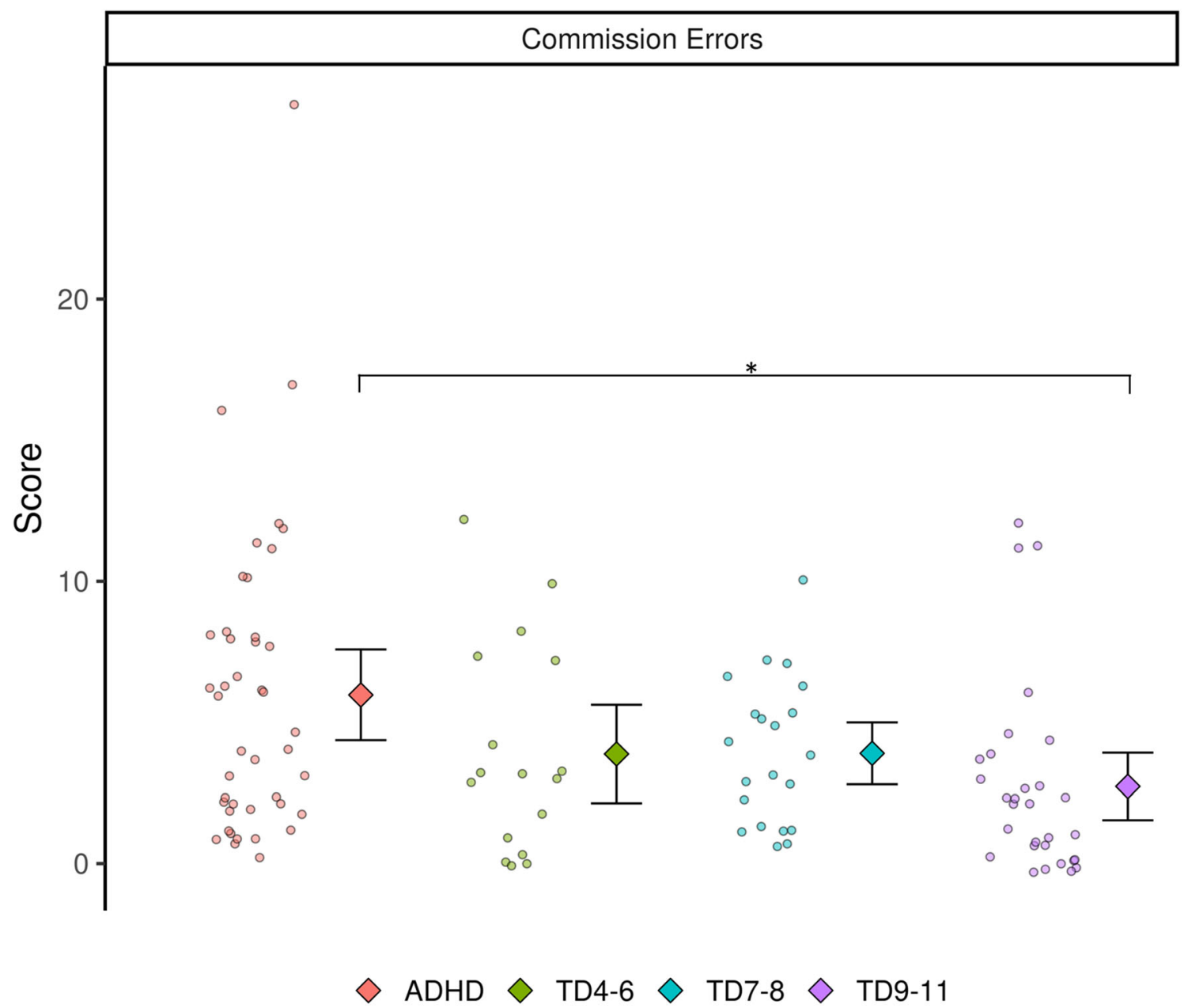

Figure 1 Go/No Go commission errors reported by group. Means, SE bars, and individual data points are plotted. Identical scores are jittered

concurrent and retrospective motor measures in Tables 4 and 5 , respectively.

Observation of Tables 4 and 5 demonstrate that statistically significant associations were observed between the two behavioural indexes of ADHD characteristics (errors on the Go/No Go task, RT variability) and the BOT2-SF fine motor measure only. This limited number of associations broadly suggests that the motor impairment in ADHD is not related to the core ADHD phenotype. The remaining analyses, as planned, were conducted by splitting the ADHD group into a low motor ability group (ADHD-L) and a high motor ability (ADHD-H) group using the zones of the BOT2-SF (Bruininks and Bruininks 2005) standard scores. The ADHD-L group had BOT2-SF standard scores within the below average and well below average zones, which indicate that performance was at least $1 \mathrm{SD}$ below the mean (i.e. $<16$ th percentile) $(N=20)$, which corresponds to the cut-off often used to indicate developmental coordination disorder (e.g. Sumner et al. 2016). The ADHD-H group had scores within the average and above average zones $(N=23)$. The details of each group are shown in Table 6. Independent $t$ tests (Bonferroni-corrected critical alpha, $p \leq 0.007$ ) revealed that the groups had similar IQ, chronological age, and ADHD symptomatology, and by design differed in motor ability. Chi-squared analysis indicated that the distribution of ADHD subtypes was similar between the two groups.

\section{The ADHD Motor Profile}

BOT2-SF MANOVA was conducted on the eight subdomains of the BOT2-SF (fine motor precision, fine motor integration, manual dexterity, bilateral coordination, balance, running speed and agility, upper limb coordination, strength) with Group as a between-participant variable (TD 4-6, TD 7-8, TD 9-11, ADHD-L, ADHD-H). This demonstrated a significant effect of group, $\mathrm{F}(32,388.816)=6.219, p<0.001$; Wilk's $\Lambda=0.218, \eta_{\mathrm{p}}{ }^{2}=0.317$. Univariate ANOVAs (Bonferroni-corrected critical alpha: $p \leq 0.00625$ ) demonstrated main effects of group for all subdomains $(p<0.00625)$ with the exception of fine motor integration $(p=0.014)$. Tukey post hoc comparisons were conducted to determine the profile of performance for the ADHD-L and ADHD-H 


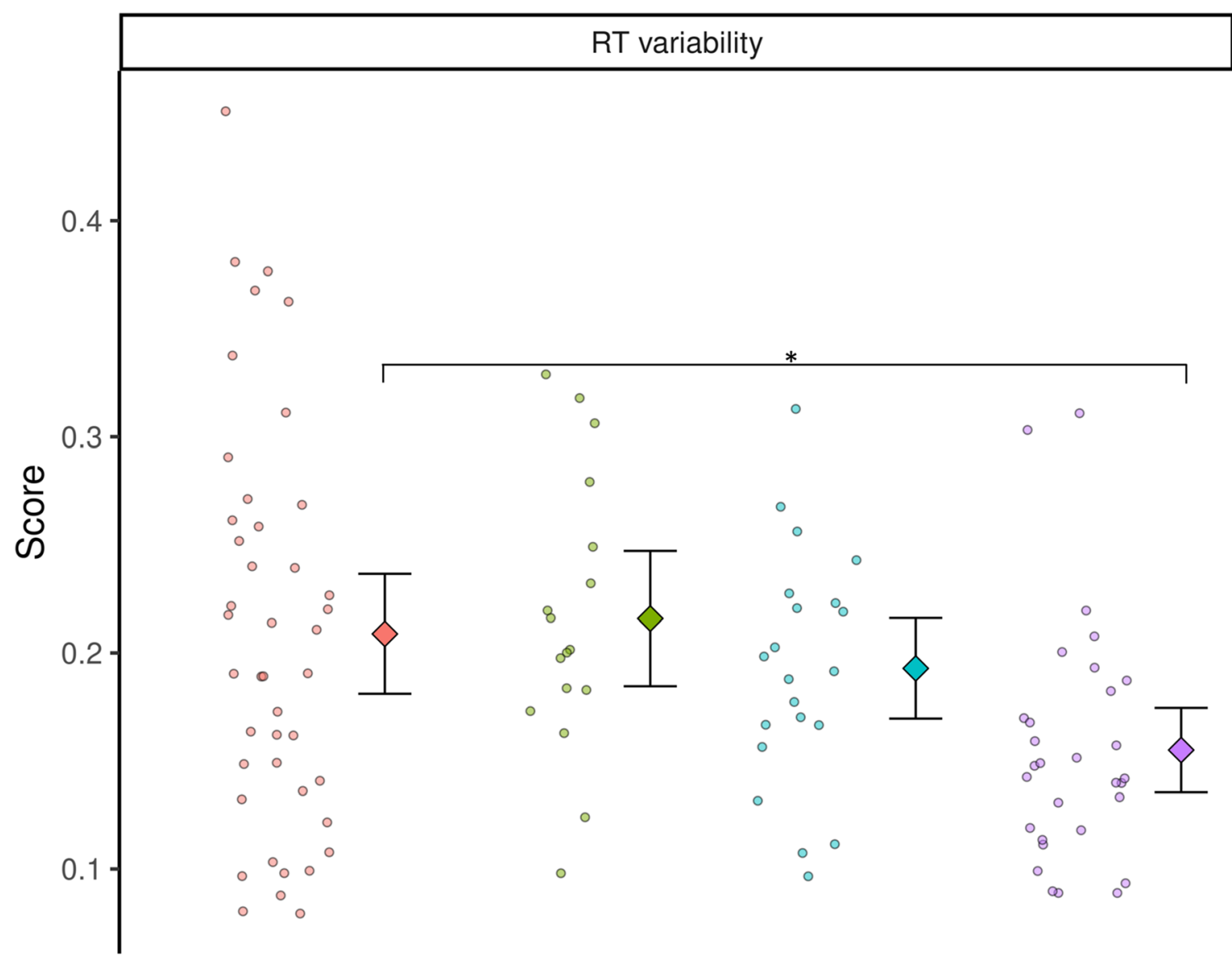

ADHD

TD4-6

TD7-8

TD9-11

Fig. 2 Go/No Go response time variability reported by group. Means, SE bars, and individual data points are plotted. Identical scores are jittered

groups. For completeness regarding profile information, this was conducted for all subdomains (including fine motor integration). The TD 9-11 group did not differ significantly in cognitive ability from either of the ADHD groups (BPVS raw score, $p=0.85$; BAS matrices ability score, $p=0.12$ ) and thus represent an approximate cognitive ability match.
Post hoc Tukey comparisons are reported here with reference to comparisons between the ADHD-H group and all other groups and between the ADHD-L group and all other groups. Significant differences across all groups are indicated on Fig. 3. Across all subdomains, the ADHD-H group was consistently at the level of the TD 9-11 group $(p>0.05$ for all

Table 4 Associations between ADHD core characteristics and BOT2-SF scores for the ADHD group (partial correlations controlling for chronological age)

\begin{tabular}{lllll}
\hline & CPRS-R:L ADHD Index & SDQ hyperactivity & Go/No Go errors & RT variability \\
\hline BOT2-SF Gross & $r=-0.19, p=0.24, d f=40$ & $r=0.26, p=0.10, d f=39$ & $r=-0.46, p=0.002, d f=40$ & $r=-0.32, p=0.04, d f=40$ \\
BOT2-SF Fine & $r=-0.07, p=0.68, d f=40$ & $r=0.05, p=0.75, d f=39$ & $r=-.49, p=0.001 *, d f=40$ & $r=-0.57, p<0.00 *^{+}, d f=40$ \\
Finger-thumb IES & $r=-0.03, p=0.87, d f=40$ & $r=0.11, p=0.49, d f=39$ & $r=.002, p=0.99, d f=40$ & $r=.310, p=0.04, d f=40$ \\
$\begin{array}{l}\text { Postural sway CoP path length } \\
\quad(\text { eyes open) }\end{array}$ & $r=0.07, p=0.65, d f=39$ & $r=-0.07, p=0.66, d f=38$ & $r=0.33, p=0.04, d f=39$ & $r=-0.06, p=0.69, d f=039$ \\
$\begin{array}{l}\text { Postural sway CoP path length } \\
\quad(\text { eyes closed) }\end{array}$ & $r=0.14, p=0.39, d f=39$ & $r=0.13, p=0.44, d f=38$ & $r=0.24, p=0.14, d f=39$ & $r=0.06, p=0.70, d f=39$ \\
\hline
\end{tabular}

$*$ denotes significance at a familywise alpha of $0.05(p \leq 0.00156) ;{ }^{+}$Note that this becomes $p=0.006$ (non-significant) when the participants with comorbid diagnoses are excluded 
Table 5 Associations between ADHD core characteristics and motor milestone month of acquisition for the ADHD group

\begin{tabular}{lllll}
\hline & CPRS-R:L ADHD Index & SDQ hyperactivity & Go/No Go errors & RT variability \\
\hline Unsupported sitting & $r=-0.51, p=0.01, N=30$ & $r=0.05, p=0.81, N=29$ & $r=-0.03, p=.88, N=30$ & $r=0.21, p=0.27, N=30$ \\
Crawling & $r=-0.26, p=0.19, N=28$ & $r=0.19, p=0.34, N=27$ & $r=0.24, p=0.23, N=28$ & $r=0.38, p=0.05, N=28$ \\
Unsupported walking & $r=0.02, p=0.90, N=36$ & $r=0.07, p=0.70, N=35$ & $r=0.39, p=0.02, N=36$ & $r=0.45, p=0.01, N=36$ \\
\hline
\end{tabular}

* denotes significance at a familywise alpha of $0.05(p \leq 0.00179)$

subdomains). They also showed no difference to the TD 7-8 group for fine motor precision, upper limb coordination, manual dexterity, and strength $(p>0.05$ for all; although note that when the participants with comorbid diagnoses were excluded ( $N=6$ ADHD-H excluded); the ADHD-H group were stronger than the TD 7-8 for manual dexterity), and no different from any TD group for fine motor integration, bilateral coordination, balance, and running speed and agility.

In contrast to the ADHD-H group, the ADHD-L group showed an uneven pattern of performance. They performed below the level of the ADHD-H group on all subdomains $(p<0.05)$ with the exception of fine motor integration, balance, and manual dexterity $(p>0.05)$. Running speed and agility was the poorest subdomain for the ADHD-L group (below the level of all TD groups, $p<0.05$ for all, although when the participants with a comorbid diagnosis were excluded ( $N=5$ ADHD-L excluded), the ADHD-L group did not differ from the TD 4-6 group for this subdomain), followed by fine motor integration, bilateral coordination, and strength (all were at the level of the TD 4-6 group only; $p>0.05$; although when the participants with a comorbid diagnosis were excluded ( $N=5$ ADHD-L excluded), the ADHD-L group did not differ from any other group for fine motor integration), then fine motor precision (at the level of the TD 4-6 and TD 7-8 group only, $p>0.05$ for both) and upper limb coordination (at the level of the TD 7-8 group only, $p>0.05$, although when the participants with a comorbid diagnosis were excluded, the ADHD-L were only marginally poorer than the ADHD-H group, $p=$ 0.09). Performance of the ADHD-L group on the manual dexterity subdomain was at the level of the TD 7-8 group and also no different from the ADHD-H group ( $p>0.05$ for both), despite being below the level of the TD 9-11 group; $p<0.05$ (although the difference between the ADHD-L group and the TD 9-11 group was not apparent when the participants with a comorbid diagnosis were excluded). Finally, for balance, the ADHD-L group did not differ from any of the TD groups or the ADHD-H group ( $p>0.05$ for all). Plots for each subdomain are shown in Fig. 3.

Motor Milestones Motor milestone data were collected from the ADHD group only. Due to the retrospective nature of this questionnaire, if parents were unsure, cells were left blank, which lead to missing data. The mean age of milestone achievement is presented in Table 7, along with the $N$ for each milestone. Six of the milestones can be compared with the World Health Organization

Table 6 Participant characteristics: ADHD-L and ADHD-H subgroups

\begin{tabular}{|c|c|c|c|c|c|}
\hline & \multicolumn{2}{|c|}{ ADHD-L $(N=20)$} & \multicolumn{2}{|l|}{ ADHD-H $(N=23)$} & \multirow[t]{2}{*}{ Group comparison } \\
\hline & $M(\mathrm{SD})$ & Range & $M(\mathrm{SD})$ & Range & \\
\hline Age & $11.74(2.25)$ & $8.01-15.63$ & $11.12(1.51)$ & $8.45-13.50$ & $p=0.28$ \\
\hline Gender $(\mathrm{m} / \mathrm{f})$ & \multicolumn{2}{|l|}{$17 / 3$} & \multicolumn{2}{|l|}{$18 / 5$} & \\
\hline BPVS-III standard score & $97.55(13.04)$ & $80-119$ & 98.96(11.09) & $82-123$ & $p=0.70$ \\
\hline BAS-III T-score & $43.45(15.01)$ & $20-74$ & $46.48(10.84)$ & $29-71$ & $p=0.45$ \\
\hline CPRS-R:L ADHD Index & $79.10(6.21)$ & $67-90$ & $76.70(8.84)$ & $61-90$ & $p=0.32$ \\
\hline CPRS-R:L Subtype & \multicolumn{2}{|c|}{$\begin{array}{l}\text { Inattentive: } N=4 \\
\text { Hyperactive-impulsive: } N=3 \\
\text { Combined: } N=7 \\
\text { * Meets neither subtype criteria: } N=6\end{array}$} & \multicolumn{2}{|c|}{$\begin{array}{l}\text { Inattentive: } N=6 \\
\text { Hyperactive-impulsive: } N=2 \\
\text { Combined: } N=6 \\
\text { * Meets neither subtype criteria: } N=9\end{array}$} & $p=0.78$ \\
\hline SDQ hyperactivity & $8.45(1.82)$ & $3-10$ & $8.91(0.97),(N=22)$ & $7-10$ & $p=0.31$ \\
\hline CBCL attention & $73.10(9.02)$ & $57-95$ & $76.84(13.28)(N=19)$ & $57-100$ & $p=0.31$ \\
\hline BOT2-SF Standard score & $36.30(3.59)$ & $28-40$ & $48.87(6.48)$ & $41-65$ & $p<0.001$ \\
\hline BOT-SF Raw scores & $58.85(10.32)$ & $38-72$ & $71.35(5.21)$ & $59-80$ & $p<0.001$ \\
\hline
\end{tabular}

*The response items used to determine CPRS-R:L subtype only partially overlap with those which comprise the ADHD Index score. As such, it is possible to score in the clinical range for the ADHD Index, but not meet criteria for either subtype 


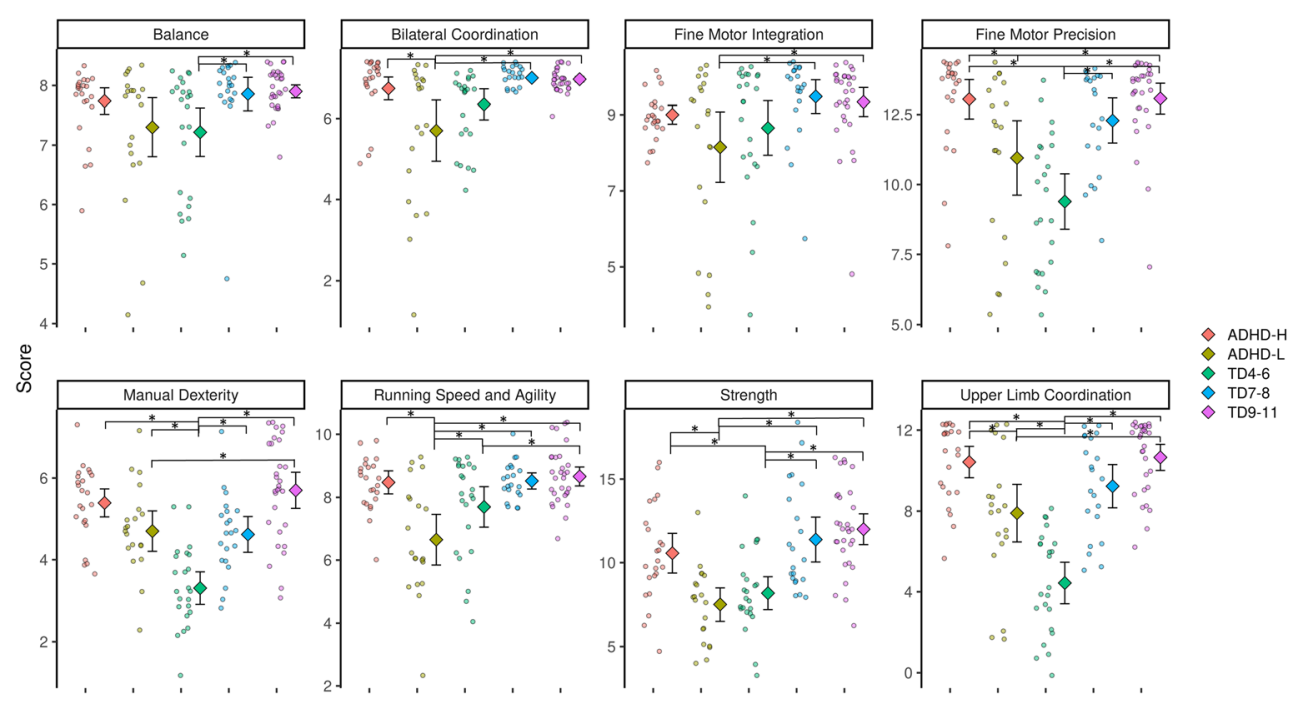

Fig. 3 BOT2-SF subdomain raw scores reported by group. Means, SE bars, and individual data points are plotted. Identical scores are jittered

(WHO) guidelines for child motor milestone achievement (Who Multicentre Growth Reference Study Group, de Onis, M 2006). For these milestones, we calculated percentiles for each group (higher percentiles represent later achievement of a milestone). Table 7 includes TD data from Sumner et al. (2016); this data were used for statistical comparison. Three ANOVAs were carried out on the month of achievement of the three milestones that were used in the correlational analyses above (sitting without support, hands and knees crawling, walking without support), each with Group (TD, ADHD-L, ADHD-H) as the betweenparticipant factor (Bonferroni correct alpha, $p \leq 0.017$ ). This showed no effect of Group for any of the milestones (sitting without support, $\mathrm{F}<1$; hands and knees crawling, $\mathrm{F}(2,58)=$ 2.12, $p=0.13, \eta_{\mathrm{p}}{ }^{2}=0.071$; walking without support, $\mathrm{F}(2$, 66) $=3.90, p=0.025, \eta_{\mathrm{p}}{ }^{2}=0.11$ ). On account of the marginal $p$ value for walking without support, Tukey paired comparisons were conducted. This demonstrated later walking in the ADHD-L group than the ADHD-H group $(p<0.05)$ but that neither ADHD group differed from the TD group $(p>0.05$ for both).

We were also interested in the predictive value of the age of motor milestone achievement on current motor ability. Correlational analyses were carried out between each motor milestone and total BOT2-SF score, for each group (TD, ADHD-L, ADHD-H). This constituted 12 correlations per group (Bonferroni-corrected critical alpha, $p \leq 0.0042)$ and revealed no significant associations ( $p>0.0042$ for all; range, $p=0.017$ to $p=0.94$ ).

Finger-Thumb Tapping Five TD participants did not complete this task. ANOVA with a between-participant factor of Group (TD4-6, TD7-8, TD9-11, ADHD-L, ADHD-H) was carried out on finger-thumb IES score. The effect of Group was significant, $\mathrm{F}(4,107)=3.38, p<0.012, \eta_{\mathrm{p}}{ }^{2}=0.11$, as shown in
Fig. 4. Tukey comparisons revealed that the ADHD-H group and the TD 9-11 group had lower IES scores than the TD 4-6 group ( $p<0.05$; all other comparisons, $p>0.05$ ), whilst the ADHD-L group did not differ from any of the other groups ( $p>0.05$ for all; although when the participants with a comorbid diagnosis were excluded, the ADHD-L group had lower IES scores than the TD $4-6$ group; $p=0.046$ ).

Postural Sway Fourteen TD datasets and one ADHD-H dataset were missing due to technical failure, whilst three TD participants did not complete the task. The final $N$ for each group was as follows: TD4 $6, N=15$; TD7-8, $N=15$; TD 9$11, N=27$; ADHD-L, $N=20$; and ADHD-H, $N=22$. ANOVA on the path length (in $\mathrm{cm}$ ) of the centre of pressure (CoP) was carried out, with a within-participant factor of condition (eyes open, eyes closed) and a between-participant factor of Group (TD4-6, TD7-8, TD 9-11, ADHD-L, ADHD$\mathrm{H})$ was conducted. There was a main effect of condition, $\mathrm{F}(1$, 94) $=63.75, p<0.001, \eta_{\mathrm{p}}{ }^{2}=0.40$ due to lower postural sway (shorter CoP path length) in the eyes open condition than the eyes closed condition. There was also a main effect of Group, $\mathrm{F}(4,94)=3.09, p=0.02, \eta_{\mathrm{p}}{ }^{2}=.12$. Tukey comparisons revealed that this was due to higher postural sway in the TD 4- to 6-year-olds than the TD 9- to 11-year-olds $(p=0.03)$ only (all other comparisons, $p>0.05$ ). There was no Group by condition interaction, $\mathrm{F}(4,94)=1.53, p=.20, \eta_{\mathrm{p}}{ }^{2}=0.06$ (Fig. 5).

\section{Discussion}

The current study had two aims: first, to investigate whether the presence of a motor deficit in ADHD is part of the ADHD phenotype or an additional impairment and, second, to 


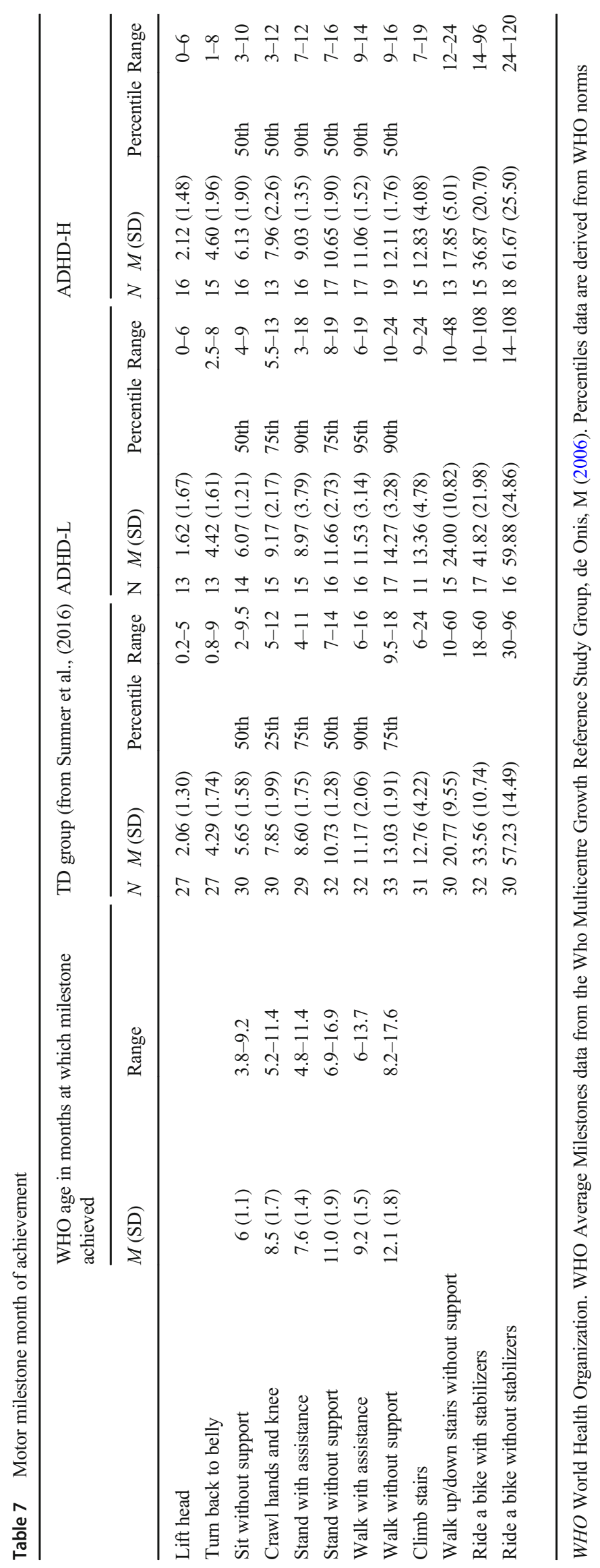


determine the motor profile of children with ADHD with reference to standardized motor tasks and tasks that have been related to cerebellum activation and how this is related to the achievement of motor milestones in early life. Our data demonstrated that twenty (47\%) out of forty-three children with ADHD had a severe motor impairment when measured using the BOT2-SF (Bruininks and Bruininks 2005). This is in line with other studies (e.g. Pitcher et al. 2003). In addition, we determined that variation in motor ability as measured by both standardized tasks (BOT2-SF) and cerebellar related tasks (postural sway and finger-thumb task performance) was not related to variation in parent report of ADHD symptoms (whilst we note limitations of parent report, this is somewhat countered by the high levels reliability of the questionnaire measures employed). However, motor performance on the BOT2-SF fine motor measure, but not BOT2-SF Gross motor, postural sway, or finger-thumb task performance, was related to a behavioural measure of inhibition and response time variability. That is, those with ADHD who made more errors (weaker inhibition) and had more variable response times for their correct responses on the Go/No Go task had lower motor scores on the BOT2-SF fine motor composite.

The relationship between fine motor ability and inhibition merits consideration of executive function (EF) (which includes inhibition; Miyake et al. 2000). EFs are impaired across many neurodevelopmental disorders such as ADHD,
ASD, and Tourette's syndrome (Johnson 2012). Johnson (2012) suggests that those with neurodevelopmental disorders who have stronger EF are more able to compensate for atypical functioning in other domains. The hypothesis that EF is acting as a protective factor, however, is not supported by our data on account of the lack of correlations between inhibition and the remaining motor measures. On a similar vein, the relatively high fine motor output demands of the Go/No Go task could be put forward to explain the relationship between RT variability (thought to measure motor output difficulties among other mechanisms, Karalunas et al. 2014) and fine motor performance on the BOT2-SF, but as above, the association does not extend to other motor tasks in our battery and so this explanation cannot be supported. Taken together, whilst our data suggest some relationship between ADHD characteristics and motor deficits, the relationship is not clear cut, and data from the majority of our measures suggest that a motor impairment is not part of the ADHD phenotype and that the presence of a clinical motor impairment is not indicative of ADHD severity.

The typical achievement of motor milestones in both ADHD-L and ADHD-H groups provides further evidence to suggest that the motor impairment is not inherent to ADHD. Furthermore, this suggests that the motor deficit present in a subset of individuals with ADHD is not the result of a primary motor deficit from infancy. Whilst we do not have information
Fig. 4 Finger-Thumb inverse efficiency scores reported by group. Means, SE bars, and individual data points are plotted. Identical scores are jittered

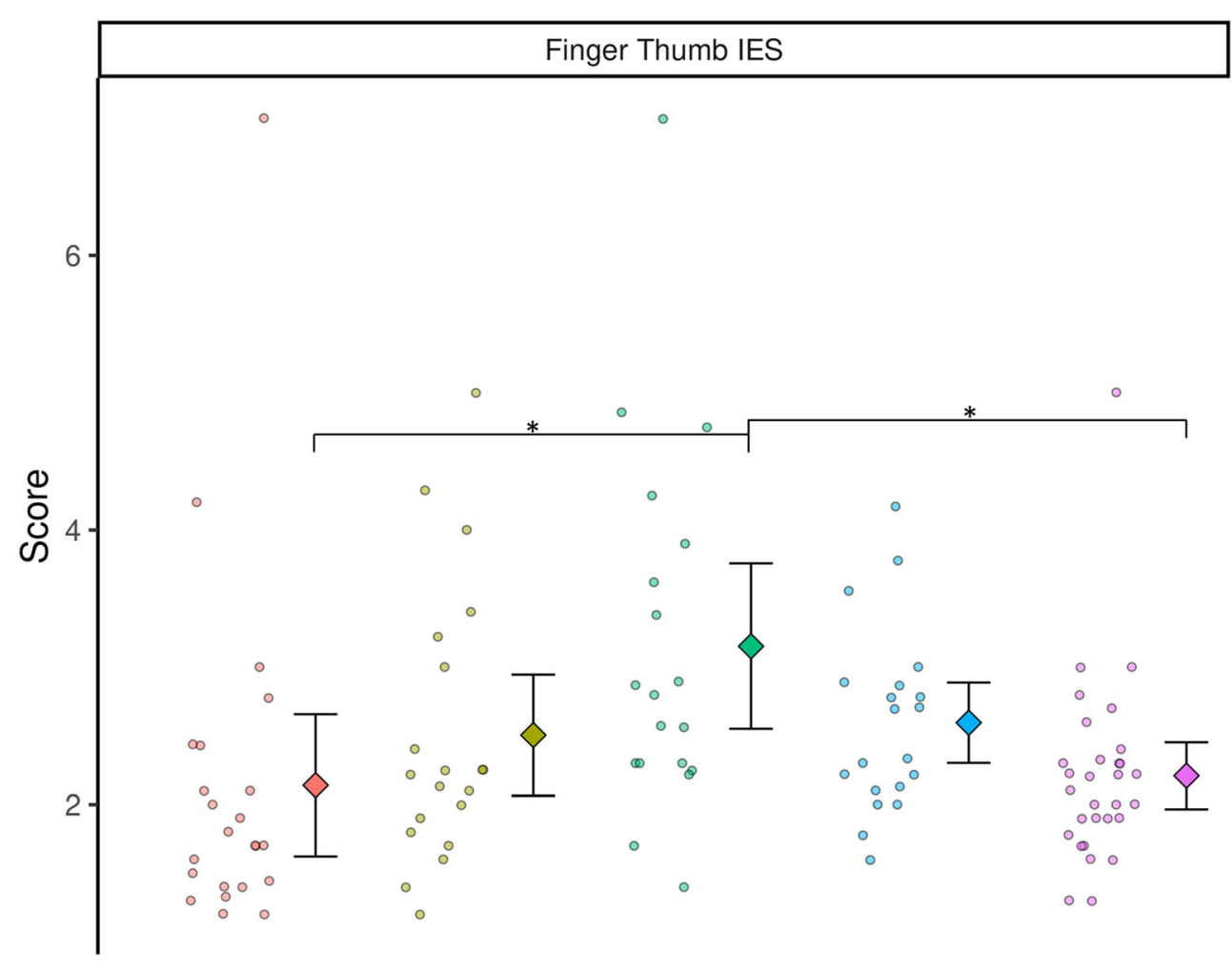

ADHD-H $\diamond A D H D-L$
TD7-8

TD9-11 


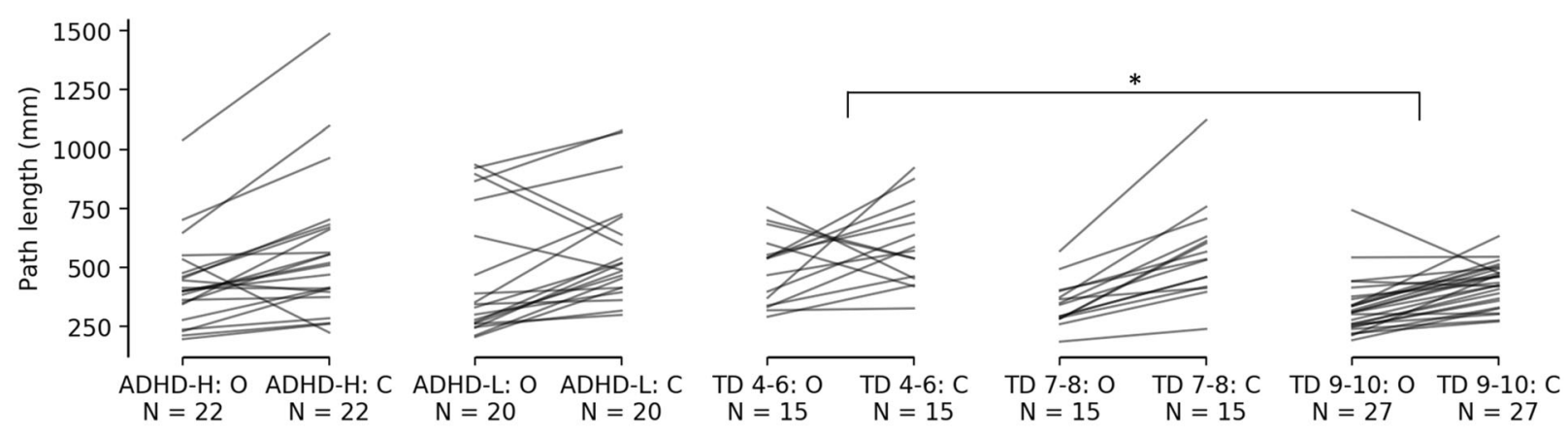

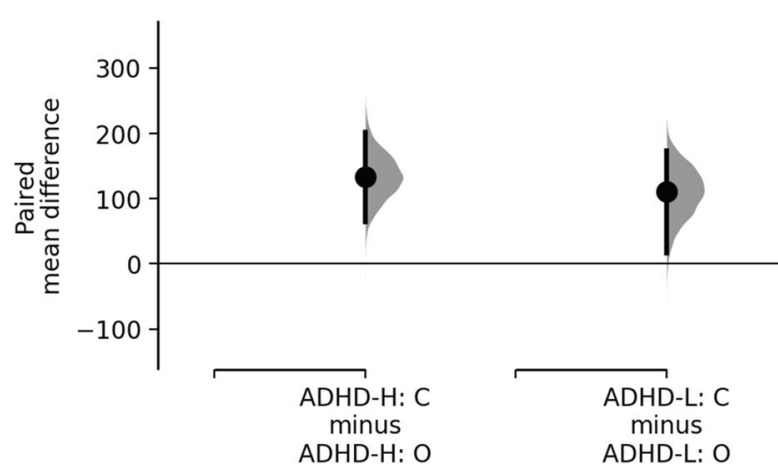

Fig. 5 Cumming estimation plot of postural sway centre of pressure $(\mathrm{CoP})$ path length (in $\mathrm{cm}$ ). The raw data are plotted on the upper axes; each paired set of observations reported by group is connected by a line. On the lower axes, each paired mean difference is plotted as a bootstrap on more fine-grained motor variables, such as motor quality in infancy in our ADHD sample, our findings suggest that the motor deficit observed in some children with ADHD becomes apparent over time, and thus, we hypothesise that this represents a cascading effect of initially more subtle impairments. Gurevitz et al. (2014) studied retrospective records of children who later obtained a diagnosis of ADHD. In line with the current study, most of the ADHD group achieved motor milestones within the typical range; although in contrast to our findings, motor development at 9 months was delayed relative to a control group and predicted subsequent diagnosis of ADHD. Furthermore, Jaspers et al. (2013) report that good gross motor ability (a high score as assessed via the Van Wiechen Scheme; Jacobusse et al. 2006) in infancy is a risk factor for ADHD symptoms. Thus, it appears that motor milestones in children with ADHD emerge within a (wide) typical timeframe (also see Athanasiadou et al. 2019 for a review).

Friedman et al. (2005) demonstrated that the relationship between attention and motor activity when looking at an object at 1-3 months is related to parent-reported attention problems at 8 years (also see Johnson et al. 2015). Similarly, D'Souza et al. (2017) reported an association between motor specialization and selective attention in 9- and 12-month-old infants. These studies demonstrate an early relationship between attention and motor domains. Thus, it is feasible that the motor deficit observed in some children with ADHD develops over time, as a product of an atypical early relationship between the attention and motor domains (Friedman et al. 2005). Equally, it is possible that the children with ADHD who do not present a motor deficit might be more resilient to overcoming these early atypicalities or that protective factors (genetic, environmental) serve to prevent or attenuate the downstream impacts of subtle early attention or motor deficits in these children. These hypotheses could be assessed by investigating the early developmental associations between attention and motor domains in children who later receive a diagnosis of ADHD.

The finding of typical motor milestone achievement in ADHD is additionally interesting, as it suggests that the motor impairment detected in a subset of children with ADHD is not DCD (for whom delayed motor milestone achievement would be predicted). This is supported by Langevin et al. (2015) who demonstrated that the cortical thinning observed in children with ADHD and DCD was not simply an additive effect of that observed in children with ADHD or children with DCD, thus demonstrating that those with co-occurring ADHD and DCD are unique, relative to their single disorder counterparts, thus lending some support to a separate DAMP disorder group (see Sonuga-Barke 2003). Typical motor milestone achievement in ADHD has strong implications for intervention with respect to the motor deficit in ADHD. Intervention with children at risk of ADHD could be 
designed to prevent or attenuate the development of a motor deficit.

For motor profile analyses, we made an a priori decision to split our ADHD group into those with a motor deficit and those without a motor deficit. Consistent with Fliers et al. (2008), these groups did not differ significantly on the questionnaire measures of ADHD characteristics, thus further supporting the conclusion above that the motor deficit is not inherent to ADHD or a marker of ADHD severity. We were then able to document the profile of motor abilities of the two groups. The ADHD-H group scored as average or above average for their age for their overall motor performance. Across the eight subdomains, they consistently performed at the level of the 9-11 TD group (who were a cognitive ability match to both ADHD groups), thus demonstrating a typical profile of motor performance. In contrast, the ADHD-L group performed in the below average and well below average range for overall motor ability for their age and presented with an uneven profile of impairment across the BOT2-SF subdomains. A particular weakness was observed for running speed and agility, which was below the level of all three of the TD groups, followed by the subdomains of bilateral coordination and strength, which were both only at the level of TD 4- to 6 -year-olds. Fine motor precision and upper limb coordination were slightly stronger, at the level of TD 7- to 8-year-olds, whilst fine motor integration, manual dexterity, and balance did not differ from the ADHD-H group. For the two tasks that are reported to activate the cerebellum, the ADHD-L and ADHD-H group did not differentiate from one another and did not show impaired performance, thus representing a relative strength within the ADHD-L motor profile.

The majority of the tasks for the weakest subdomains of the ADHD-L group involved high physical effort (e.g. hopping, jumping, push-ups). Differences in motor familiarity have also been shown to relate to variation in brain activation (Plata Bello et al. 2015). We ruled out both variation in motor familiarity and/or poor physical fitness (as a result of low motivation to be physically active) as an explanation for the motor profile; these explanations cannot account for the whole motor profile, neither do they offer a syndrome-specific explanation for the motor profile observed.

As noted in the introduction, the few previous studies that have documented the motor profile in ADHD do not report consistent findings. Previous studies are also hard to interpret as we do not know how many of their sample had a general motor impairment; our findings suggest that the inclusion of children without a general motor impairment dilutes the profiles reported in previous studies. Manual dexterity has been reported as a specific deficit by Pitcher et al. (2003) and Brossard-Racine et al. (2012), but not by Tseng et al. (2004) or Çak et al. (2018). Our manual dexterity data align with Tseng et al. (2004) and Çak et al. (2018) who also used the BOT and thus suggest that differences across studies might be task related. Tseng et al. (2004) and Çak et al. (2018) report deficits in balance, whilst Pitcher et al. (2003) and BrossardRacine et al. (2012) did not detect any specific impairments in balance in ADHD; our data show that balance was not impaired on the subtest of the BOT2-SF or on our postural sway task. Observation of Fig. 3 suggests that any group differences in the balance subdomain of the BOT2-SF might be masked by the high proportion of ceiling scores across groups for this subdomain. This is surprising because the BOT2 is purported to be suitable up to the level of a 21-year-old. The postural sway task is arguably a more sensitive measure of balance. The four previous studies that have employed measures of postural sway, in contrast to our findings, consistently report deficits in performance in children and adults with ADHD (Bucci et al. 2014, 2017; Hove et al. 2015; Shorer et al. 2012). The differentiation between eyes open and eyes closed conditions in our task demonstrate that the task is sensitive. However, technical failure accounted for a lot of missing data in our sample, and so, we cannot rule out a lack of power as an explanation for out null finding with respect to group.

\section{Limitations and Future Research}

Our study is limited by the sample size of our ADHD group, which restricts the power of analyses, particularly the associational analyses. This is also a consideration on the few occasions when the results differ when the participants with comorbid diagnoses are excluded; one cannot determine whether these differences are due to reduced power or sample characteristics. Observation of Fig. 3 also demonstrates the large intra-group variability for many of the motor measures of the BOT2-SF. Even within the ADHD-L group, for most subdomains, the scores range from the ceiling score for the subdomain to below even the youngest TD children. This also highlights intraparticipant variability. These participants met the criteria of falling below the 16th percentile overall, thus their high scores on one subdomain are necessarily countered by low scores in other subdomains. This not only emphasizes the uneven profile but also highlights that whilst we (and others) report a group level ADHD motor profile, this profile is subject to wide intra-group variability and that the differences across studies might be explained by the large heterogeneity in the (small) samples. A larger sample would give more confidence in the ADHD motor profile at the group level.

Our data are also cross-sectional. Whilst this is an ideal design for determining the ADHD motor profile for the agerange employed, the use of cross-sectional data alone to determine the association with ADHD core characteristics is a limitation. The inclusion of motor milestone data provides some developmental insight, but to date there are no longitudinal developmental studies that have addressed this question. Familial data is also pertinent to this question; as discussed, Fliers et al. (2008) used familial data to also conclude that a 
motor impairment is not part of the ADHD phenotype. Future studies could include longitudinal data and familial data in the same study.

Our data suggest that the motor deficit observed in a large proportion of children with ADHD is not part of the ADHD phenotype. This is based on the lack of significant correlations between the parent report questionnaires of ADHD phenotypic characteristics and empirical measures of motor performance and no difference in ADHD symptom scores between the ADHD-H and ADHD-L group. Of strong interest to future research is the typical achievement of motor milestones in the ADHD-L group. Whilst this was evaluated using retrospective parent report, comparison with Sumner et al. (2016) - who used the same measure with children with DCD - adds validity to the approach. We hypothesise that the motor deficit observed in some children with ADHD is a result of subtle impairments (perhaps in the attention or motor domain) in infancy, which impact motor development. Future research is required to address this hypothesis.

Data Availability Statement All data and SPSS syntax are available at the Open Science Framework (https://osf.io/nfzw8/).

Authors' Contributions EF, AKS, and EH conceived of the initial study design, with later contributions from HDS. HDS and LM collected the data from typically developing participants. $\mathrm{AB}$ recruited and collected data from the ADHD participants and wrote-up initial Introduction and Methods sections of the paper. EF wrote the final manuscript with consultation from EH. ES contributed the TD data for the motor milestone measure and final manuscript formatting. DB assisted with coding the Wii-board data, provided general advice regarding the use of the Wiiboard, and provided Figs. 1 to 4 . We sadly state that AKS passed away before this study was completed. We would like to publicly acknowledge the influence of her thinking and theoretical views in writing up this study. All authors read and approved the final manuscript.

Funding Information This research was supported by The Waterloo Foundation (Grant number: FPS 596) and an ESRC PhD studentship to LM. HD is a Beatrice Mary Dale Research Fellow supported by Newnham College, University of Cambridge. Thank you to Fatima Esfandi for help with data collection. Thank you to Abacus Belsize Primary School, William Patten Primary school, Islington ADHD support group, ADHD Richmond and Kingston, and the ADHD Foundation UK for enabling this research to take place. Special thanks go to the participants for taking part in the study. Annette Karmiloff-Smith is sadly no longer with us. Annette was very involved in the conception of this study, and her ideas are present throughout the manuscript. We thank her for her contributions to this research, even though she was unable to see the end product.

\section{Compliance with Ethical Standards}

Conflict of Interest On behalf of all authors, the corresponding author states that there is no conflict of interest.

Ethics Statement This study has been through the ethics review process at UCL Institute of Education, University of London and has been approved.
Open Access This article is licensed under a Creative Commons Attribution 4.0 International License, which permits use, sharing, adaptation, distribution and reproduction in any medium or format, as long as you give appropriate credit to the original author(s) and the source, provide a link to the Creative Commons licence, and indicate if changes were made. The images or other third party material in this article are included in the article's Creative Commons licence, unless indicated otherwise in a credit line to the material. If material is not included in the article's Creative Commons licence and your intended use is not permitted by statutory regulation or exceeds the permitted use, you will need to obtain permission directly from the copyright holder. To view a copy of this licence, visit http://creativecommons.org/licenses/by/4.0/.

\section{References}

Achenbach, T., Rescorla, L. (2001). Manual for the ASEBA school-age forms and profiles: An integrated system of multi-informant assessment. ASEBA Burlington.

American Psychiatric Association (2013). Diagnostic and Statistical Manual of Mental Disorders, (5 ${ }^{\text {th }}$ ed.). https://doi.org/10.1176/ appi.books.9780890425596

Athanasiadou, A., Buitelaar, J. K., Brovedani, P., Chorna, O., Fulceri, F., Guzzetta, A., \& Scattoni, M. L. (2019). Early motor signs of attention-deficit hyperactivity disorder: A systematic review. European Child \& Adolescent Psychiatry, 1-14. https://doi.org/10. 1007/s00787-019-01298-5.

Benda, B. J., Riley, P. O., \& Krebs, D. E. (1994). Biomechanical relationship between center of gravity and center of pressure during standing. IEEE Transactions on Rehabilitation Engineering, 2(1), 3-10. https://doi.org/10.1109/86.296348.

Brossard-Racine, M., Shevell, M., Snider, L., Bélanger, S. A., \& Majnemer, A. (2012). Motor skills of children newly diagnosed with attention deficit hyperactivity disorder prior to and following treatment with stimulant medication. Research in Developmental Disabilities, 33, 2080-2087. https://doi.org/10.1016/j.ridd.2012.06. 003.

Brouwer, S. I., Beijsterveldt, T. C. E. M., van Bartels, M., Hudziak, J. J., \& Boomsma, D. I. (2006). Influences on achieving motor milestones: A twin-singleton study. Twin Research and Human Genetics, 9, 424-430. https://doi.org/10.1375/twin.9.3.424.

Bruininks, R. H. (1978). Bruininks-Oseretsky test of motor proficiency. American Guidance Service.

Bruininks, B. D., \& Bruininks, R. H. (2005). Bruininks-Oseretsky test of motor proficiency (2nd). Pearson Assessment.

Bucci, M. P., Seassau, M., Larger, S., Bui-Quoc, E., \& Gerard, C.-L. (2014). Effect of visual attention on postural control in children with attention-deficit/hyperactivity disorder. Research in Developmental Disabilities, 35, 1292-1300. https://doi.org/10.1016/j.ridd.2014.03. 029.

Bucci, M. P., Goulème, N., Stordeur, C., Acquaviva, E., Septier, M., Lefebvre, A., Peyre, H., \& Delorme, R. (2017). Motor impairment in children with autistic spectrum disorder and in children with attention deficit hyperactivity disorder. Journal of Mental Health Care, 1, 4-8.

Çak, H. T., Karaokur, R., Uysal, S. A., Artik, A., Kabak, V. Y., Karakök, B., Sahan, N., Karaer, Y., Karabucak, B., Özusta, S., \& Kültür, E. Ç. (2018). Motor proficiency in children with attention deficit hyperactivity disorder: Associations with cognitive skills and symptom severity. Türkish Journal of Psychiatry, 29(2), 1-10. https://doi.org/ $10.5080 / \mathrm{u} 22884$.

Clark, J. E., \& Metcalfe, J. S. (2002). The mountain of motor development: A metaphor. In J. E. Clark \& J. H. Humphrey (Eds.), Motor 
development: Research and reviews (Vol. 2, pp. 163-190). National Association for Sport and Physical Education.

Clark, R. A., Bryant, A. L., Pua, Y., McCrory, P., Bennell, K., \& Hunt, M. (2010). Validity and reliability of the Nintendo Wii balance board for assessment of standing balance. Gait \& Posture, 31, 307-310. https://doi.org/10.1016/j.gaitpost.2009.11.012.

Clearfield, M. W. (2011). Learning to walk changes infants' social interactions. Infant Behavior and Development, 34, 15-25. https://doi. org/10.1016/j.infbeh.2010.04.008.

Conners, C. K. (1997). Conners' parent rating scale-revised (L). MultiHealth Systems.

Conners, C. K. (2003). Conners' rating scales-revised: Technical manual. Multi-Health Systems.

Conners, C. K., Sitarenios, G., Parker, J. D. A., \& Epstein, J. N. (1998). The revised Conners' parent rating scale (CPRS-R): Factor structure, reliability, and criterion validity. Journal of Abnormal Child Psychology, 26, 257-268. https://doi.org/10.1023/A: 1022602400621.

D'Souza, H., Cowie, D., Karmiloff-Smith, A., \& Bremner, A. J. (2017). Specialization of the motor system in infancy: From broad tuning to selectively specialized purposeful actions. Developmental Science, 20(4), e12409. https://doi.org/10.1111/desc.12409.

Dunn, L. M., \& Dunn, D. M. (2009). The British picture vocabulary scale. GL Assessment Limited.

Elliot, C. D., \& Smith, P. (2011). British ability scales 3rd ed. (BAS-3). GL Assessment.

Epstein, J. N., Langberg, J. M., Rosen, P. J., Graham, A., Narad, M. E., Antonini, T. N., Brinkman, W. B., Froehlich, T., Simon, J. O., \& Altaye, M. (2011). Evidence for higher reaction time variability for children with $\mathrm{ADHD}$ on a range of cognitive tasks including reward and event rate manipulations. Neuropsychology, 25(4), 427-441. https://doi.org/10.1037/a0022155.

Fawcett, A. J., Nicolson, R. I., \& Dean, P. (1996). Impaired performance of children with dyslexia on a range of cerebellar tasks. Annals of Dyslexia, 46, 259-283. https://doi.org/10.1007/BF02648179.

Flatters, I., Culmer, P., Holt, R. J., Wilkie, R. M., \& Mon-Williams, M. (2014). A new tool for assessing head movements and postural sway in children. Behavior Research Methods, 46, 950-959. https://doi. org/10.3758/s13428-013-0419-x.

Fliers, E., Vermeulen, S., Rijsdijk, F., Altink, M., Buschgens, C., Rommelse, N., Faraone, S., Sergeant, J., Buitelaar, J., \& Franke, B. (2008). ADHD and poor motor performance from a family genetic perspective. Journal of the American Academy of Child and Adolescent Psychiatry, 48, 25-34. https://doi.org/10.1097/CHI. 0b013e31818b1ca2.

Friedman, A. H., Watamura, S. E., \& Robertson, S. S. (2005). Movement-attention coupling in infancy and attention problems in childhood. Developmental Medicine and Child Neurology, 47(10), 660-665. https://doi.org/10.1017/S0012162205001350.

Gillberg, C. (2003). Deficits in attention, motor control, and perception: A brief review. Archives of Disease in Childhood, 88(10), 904-910. https://doi.org/10.1136/adc.88.10.904.

Goodman, R. (1997). The strengths and difficulties questionnaire: A research note. Journal of Child Psychology and Psychiatry, 38, 581586. https://doi.org/10.1111/j.1469-7610.1997.tb01545.x.

Goodman, R. (2001). Psychometric properties of the strengths and difficulties questionnaire. Journal of the American Academy of Child \& Adolescent Psychiatry, 40, 1337-1345.

Goulardins, J. B., Marques, J. C. B., \& De Oliveira, J. A. (2017). Attention deficit hyperactivity disorder and motor impairment: A critical review. Perceptual and Motor Skills, 124(2), 425-440. https://doi.org/10.1177/0031512517690607.

Gurevitz, M., Geva, R., Varon, M., \& Leitner, Y. (2014). Early markers in infants and toddlers for development of ADHD. Journal of Attention Disorders, 18, 14-22. https://doi.org/10.1177/1087054712447858.
Henderson, S. E., \& Sugden, D. A. (1992). Movement assessment battery for children. The Psychological Corporation.

Henderson, S. E., Sugden, D. A., \& Barnett, A. L. (2007). Movement assessment battery for children (2nd ed.). The Psychological Corporation.

Hove, M. J., Zeffiro, T. A., Biederman, J., Li, Z., Schmahmann, J., \& Valera, E. M. (2015). Postural sway and regional cerebellar volume in adults with attention-deficit/hyperactivity disorder. NeuroImage: Clinical, 8, 422-428. https://doi.org/10.1016/j.nicl.2015.05.005.

Jacobusse, G., Van Buuren, S., \& Verkerk, P. H. (2006). An interval scale for development of children aged 0-2 years. Statistics in Medicine, 25(13), 2272-2283. https://doi.org/10.1002/sim.2351.

Jaspers, M., de Winter, A. F., Buitelaar, J. K., Verhulst, F. C., Reijneveld, S. A., \& Hartman, C. A. (2013). Early childhood assessments of community pediatric professionals predict autism spectrum and attention deficit hyperactivity problems. Journal of Abnormal Child Psychology, 41, 71-80. https://doi.org/10.1007/s10802-012-96534.

Johnson, M. H. (2012). Executive function and developmental disorders: The flip side of the coin. Trends in Cognitive Sciences, 16(9), 454 457. https://doi.org/10.1016/j.tics.2012.07.001.

Johnson, M. H., Gliga, T., Jones, E., \& Charman, T. (2015). Annual research review: Infant development, autism, and ADHD-Early pathways to emerging disorders. Journal of Child Psychology and Psychiatry, 56(3), 228-247. https://doi.org/10.1111/jcpp.12328.

Johnston, L., \& Watter, P. (2006). Movement assessment battery for children. The Australian Journal of Physiotherapy, 52, 68.

Kaiser, M.-L., Schoemaker, M. M., Albaret, J.-M., \& Geuze, R. H. (2015). What is the evidence of impaired motor skills and motor control among children with attention deficit hyperactivity disorder (ADHD)? Systematic review of the literature. Research in Developmental Disabilities, 36, 338-357. https://doi.org/10.1016/j. ridd.2014.09.023.

Karalunas, S. L., Geurts, H. M., Konrad, K., Bender, S., \& Nigg, J. T. (2014). Reaction time variability in ADHD and autism spectrum disorders: Measurement and mechanisms of a proposed transdiagnostic phenotype. Journal of Child Psychology and Psychiatry, 55(6), 685-710. https://doi.org/10.1111/jcpp.12217.

Kim, B.-N., Lee, J.-S., Shin, M.-S., Cho, S.-C., \& Lee, D.-S. (2002). Regional cerebral perfusion abnormalities in attention deficit/ hyperactivity disorder. European Archives of Psychiatry and Clinical Neuroscience, 252, 219-225. https://doi.org/10.1007/ s00406-002-0384-3.

Kretch, K. S., Franchak, J. M., \& Adolph, K. E. (2014). Crawling and walking infants see the world differently. Child Development, 85, 1503-1518. https://doi.org/10.1111/cdev.12206.

Langevin, L. M., Macmaster, F. P., \& Dewey, D. (2015). Distinct patterns of cortical thinning in concurrent motor and attention disorders. Developmental Medicine and Child Neurology, 57(3), 257-264. https://doi.org/10.1111/dmen.12561.

Lavasani, N. M., \& Stagnitti, K. (2011). A study on fine motor skills of Iranian children with attention deficit/hyper activity disorder aged from 6 to 11 years. Occupational Therapy International, 18, 106114. https://doi.org/10.1002/oti.306.

Leach, J. M., Mancini, M., Peterka, R. J., Hayes, T. L., \& Horak, F. B. (2014). Validating and calibrating the Nintendo Wii balance board to derive reliable Center of Pressure Measures. Sensors (Basel, Switzerland), 14(10), 18244-18267. https://doi.org/10.3390/ s141018244.

Miyake, A., Friedman, N., Emerson, M., Witzki, A., \& Howerter, A. (2000). The unity and diversity of executive functions and their contributions to complex "frontal lobe" tasks: A latent variable analysis. Cognitive Psychology, 41, 49-100.

Mokobane, M., Pillay, B. J., \& Meyer, A. (2019). Fine motor deficits and attention deficit hyperactivity disorder in primary school children. 
South African Journal of Psychiatry, 25, a1232. https://doi.org/10. 4102/sajpsychiatry.v25i0.1232.

Mostofsky, S. H., Reiss, A. L., Lockhart, P., \& Denckla, M. B. (1998). Evaluation of cerebellar size in attention-deficit hyperactivity disorder. Journal of Child Neurology, 13, 434-439. https://doi.org/10. 1177/088307389801300904.

Mostofsky, S. H., Rimrodt, S. L., Schafer, J. G. B., Boyce, A., Goldberg, M. C., Pekar, J. J., \& Denckla, M. B. (2006). Atypical motor and sensory cortex activation in attention-deficit/hyperactivity disorder: A functional magnetic resonance imaging study of simple sequential finger tapping. Biological Psychiatry, 59, 48-56. https://doi.org/10. 1016/j.biopsych.2005.06.011.

Nigg, J. T. (1999). The ADHD response inhibition deficit as measured by the stop task: Replication with DSM-IV combined type, extension, and qualification. Journal of Abnormal Child Psychology, 27, 391400. https://doi.org/10.1023/A:1021980002473.

Nigg, J. T., Willcutt, E. G., Doyle, A. E., \& Sonuga-Barke, E. J. (2005). Causal heterogeneity in attention-deficit/ hyperactivity disorder: Do we need neuropsychologically impaired subtypes? Biological Psychiatry, 57(11), 1224-1230.

Piek, J. P., Dyck, M. J., Nieman, A., Anderson, M., Hay, D., Smith, L. M., \& Hallmayer, J. (2004). The relationship between motor coordination, executive functioning and attention in school aged children. Archives of Clinical Neuropsychology, 19, 1063-1076. https://doi.org/10.1016/j.acn.2003.12.007.

Pitcher, T. M., Piek, J. P., \& Hay, D. A. (2003). Fine and gross motor ability in males with ADHD. Developmental Medicine and Child Neurology, 45, 525-535. https://doi.org/10.1017/ S0012162203000975.

Plata Bello, J. P., Modroño, C., Marcano, F., \& González-Mora, J. L. (2015). The effect of motor familiarity during simple finger opposition tasks. Brain Imaging and Behavior, 9, 828-838. https://doi.org/ 10.1007/s11682-014-9340-x.

Polanczyk, G., Lima, M. S., Horta, B. L., Biederman, J., \& Rohde, L. A. (2007). The worldwide prevalence of ADHD: A systematic review and metaregression analysis. American Journal of Psychiatry, 164(6), 942-948. https://doi.org/10.1176/ajp.2007.164.6.942.

Purser, H. R. M., Farran, E. K., Courbois, Y., Lemahieu, A., Sockeel, P., Mellier, D., \& Blades, M. (2015). The development of route learning in down syndrome, Williams syndrome and typical development: Investigations with virtual environments. Developmental Science, 18, 599-613. https://doi.org/10.1111/desc. 12236 .

Racine, M., Majnemer, A., Shevel, M., \& Snider, L. (2008). Handwriting performance in children with attention deficit hyperactivity disorder (ADHD). Journal of Child Neurology, 23, 399-406. https://doi.org/ 10.1177/0883073807309244.

Rosch, K. S., Dirlikov, B., \& Mostofsky, S. H. (2013). Increased intrasubject variability in boys with ADHD across tests of motor and cognitive control. Journal of Abnormal Child Psychology, 41, 485-495. https://doi.org/10.1007/s10802-012-9690-z.

Scharoun, S. M., Bryden, P. J., Otipkova, Z., Musalek, M., \& Lejcarova, A. (2013). Motor skills in Czech children with attention-deficit/hyperactivity disorder and their neurotypical counterparts. Research in Developmental Disabilities, 34, 4142-4153. https://doi.org/10. 1016/j.ridd.2013.08.011.

Shorer, Z., Becker, B., Jacobi-Polishook, T., Oddsson, L., \& Melzer, I. (2012). Postural control among children with and without attention deficit hyperactivity disorder in single and dual conditions. European Journal of Pediatrics, 171, 1087-1094. https://doi.org/ 10.1007/s00431-012-1695-7.

Sonuga-Barke, E. J. S. (2003). On the intersection between AD/ HD and DCD: The DAMP hypothesis. Child and Adolescent Mental Health, 8, 114-116.

Sumner, E., Leonard, H. C., \& Hill, E. L. (2016). Overlapping phenotypes in autism Spectrum disorder and developmental coordination disorder: A cross-syndrome comparison of motor and social skills. Journal of Autism and Developmental Disorders, 46, 2609-2620. https://doi.org/10.1007/s10803-016-2794-5.

Sumner, E., Hutton, S. B., Kuhn, G., \& Hill, E. L. (2018). Oculomotor atypicalities in developmental coordination disorder. Developmental Science, 21, e12501. https://doi.org/10.1111/desc.12501.

Tseng, M. H., Henderson, A., Chow, S. M., \& Yao, G. (2004). Relationship between motor proficiency, attention, impulse, and activity in children with ADHD. Developmental Medicine and Child Neurology, 46, 381-388. https://doi.org/10.1017/ S0012162204000623.

Tye, C., Johnson, K. A., Kelly, S. P., Asherson, P., Kuntsi, J., Ashwood, K. L., Azadi, B., Bolton, P., \& McLoughlin, G. (2016). Response time variability under slow and fast-incentive conditions in children with ASD, ADHD and ASD plus ADHD. Journal of Child Psychology and Psychiatry, 57(12), 1414-1423. https://doi.org/10. $1111 /$ jcpp. 12608.

Who Multicentre Growth Reference Study Group, de Onis, M. (2006). WHO motor development study: Windows of achievement for six gross motor development milestones. Acta Paediatrica, 95, 86-95. https://doi.org/10.1111/j.1651-2227.2006.tb02379.x.

Ziereis, S., \& Jansen, P. (2016). Correlation of motor abilities and executive functions in children with ADHD. Applied Neuropsychology: Child, 5, 138-148. https://doi.org/10.1080/21622965.2015. 1038746.

Publisher's Note Springer Nature remains neutral with regard to jurisdictional claims in published maps and institutional affiliations. 